\title{
Broadening open access: sharing early outcomes in geoscience
}

\section{JEROEN BOSMAN}

Utrecht University

Presenting Author: j.bosman@uu.nl

While open access to formally published outputs is important, it leaves quite some potential for early reuse, feedback and collaboration unused. Sharing of early outputs, from proposals, data, workflows, protocols, code, posters and presentations to preprints can all be considered part of open science. In many scientific fields, including geoscience, sharing of early outputs has become part of the research practice. Many tools and platforms support these practices using accepted standards and sustainable models. There is incomplete but increasing evidence of the added value of sharing early outputs, at researcher level as well as at science system level. There are however also still serious barriers holding researchers back. An exploration of current practices as well as community norms and disciplinary culture is used uncover these barriers and suggest ways to lower them. Each type of output has its own combination of opportunities of sharing, barriers to sharing, technologies that support sharing and research community norms that accommodate or hinder the adoption of sharing practices.

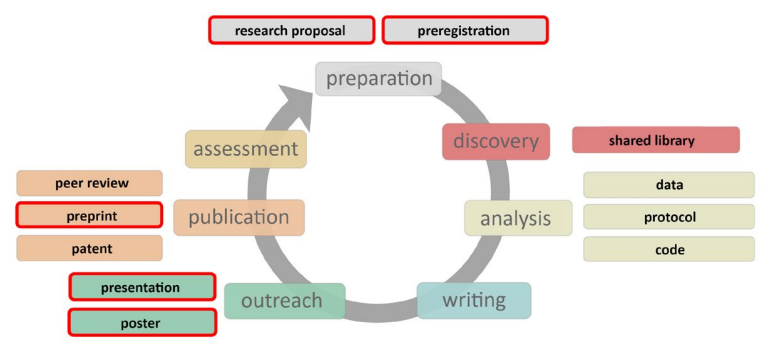

